\title{
Diabetes and Chronic Pancreatitis: Considerations in the Holistic Management of an Often Neglected Disease
}

\author{
Philip C. Johnston (D, ${ }^{1}$ Judith Thompson, ${ }^{2}$ Allison Mckee, ${ }^{2}$ Connor Hamill, ${ }^{1}$ and Ian Wallace ${ }^{1}$ \\ ${ }^{1}$ Diabetes Department, 51 Lisburn Road, BT9 7AB Belfast City Hospital, UK \\ ${ }^{2}$ Dietetics Department, 51 Lisburn Road, BT9 7AB Belfast City Hospital, UK
}

Correspondence should be addressed to Philip C. Johnston; philip.johnston@belfasttrust.hscni.net

Received 30 April 2019; Revised 13 August 2019; Accepted 11 September 2019; Published 7 October 2019

Guest Editor: Amy L. Hui

Copyright (C) 2019 Philip C. Johnston et al. This is an open access article distributed under the Creative Commons Attribution License, which permits unrestricted use, distribution, and reproduction in any medium, provided the original work is properly cited.

\begin{abstract}
Diabetes secondary to chronic pancreatitis (CP) or type 3cDM refers to a brittle form of diabetes and is often characterised by hypoglycaemic episodes, erratic glycaemic control, and impaired quality of life. It differs from other forms of diabetes and is typically characterised by concurrent pancreatic endocrine and exocrine insufficiency which can present as malabsorption and nutritional deficiencies. In this review, we discuss the pathogenesis, epidemiology, and the practicalities of diagnosis, screening, and management of this condition.
\end{abstract}

\section{Clinical Case}

Mr. $\mathrm{G}$ is a 46-year-old male who was diagnosed with chronic pancreatitis (CP) 6 years prior to his outpatient clinic consultation. He had developed diabetes mellitus a year after his CP was diagnosed. He has a history of partial pancreatectomy due to pancreatic pseudocyst formation 4 years prior. The aetiology of his $\mathrm{CP}$ was from alcohol dependence. His last episodes of pancreatitis was in the previous year. His $\mathrm{HbAlc}$ is $75 \mathrm{mmol} / \mathrm{mol}$, and he is maintained on a basal bolus regimen of Lantus and NovoRapid. His ACR and lipids are at target. His recent eye and feet screening testing is normal. He takes 50,000 units of Creon with his meals. He smokes 20 cigarettes per day and has been referred for smoking cessation. He has stopped alcohol one month prior to his clinic visit. He continues to experience intermittent abdominal pain and loose stools. He has had a weight loss of one stone over the previous 3 months due to inadequate glycaemic control and intermittent compliance with his Creon (PERT-pancreatic enzyme replacement therapy). After discussion with the patient regarding the benefits of compliance of his medications and the rationale for the importance of good glycaemic control, he reported a steady improvement in his quality of life. At a follow-up 6 months later and with improved compliance with PERT and engagement with dietary changes and titration of his insulin regimen, he has gained $5 \mathrm{~kg}$ with improvement in his gastrointestinal symptoms; glycaemic control has improved to a HbAlc of $67 \mathrm{mmol} / \mathrm{mol}$.

\section{Introduction}

Pancreatogenic diabetes or type $3 c$ diabetes mellitus $(\mathrm{T} 3 \mathrm{cDM})$ refers to diabetes arising from pancreatic disease and is characterised by pancreatic exocrine insufficiency (PEI). The prevalence of T3cDM is around $5-10 \%$ of diabetic populations with the majority $(80 \%)$ arising from chronic pancreatitis (CP) [1]. Other common aetiologies of pancreatogenic diabetes include cystic fibrosis, haemochromatosis, pancreatic cancer, and pancreatectomy [2]. Common aetiologies of chronic pancreatitis include alcohol, gallstones, genetic (PRSS1, SPINK1, and CFTR mutations), and autoimmune [3]. The pancreas in chronic pancreatitis is characterised by an inflammatory response with infiltration of inflammatory cells and release of proinflammatory cytokines and activation of pancreatic stellate cells with synthesis of extracellular matrix proteins and fibrogenesis; the resulting fibrosis leads to progressive and eventual exocrine and 
TABle 1: Proposed diagnostic criteria for type $3 c$ diabetes mellitus.

Major criteria

(i) Presence of exocrine pancreatic insufficiency (faecal elastase)

(ii) Pathological pancreatic imaging (endoscopic ultrasound (EUS), MRI, and CT)

(iii) No type 1 diabetes mellitus-associated autoimmune markers Minor criteria

(i) The absence of pancreatic polypeptide (PP) secretion

(ii) Impaired incretin secretion

(iii) No excessive insulin resistance (HOMA-IR)

(iv) Impaired beta cell function (HOMA-B, C-peptide/glucoseratio)

(v) Low serum levels of lipid soluble vitamins (A, D, E, and K)

endocrine cell destruction [4]. Exocrine insufficiency typically presents earlier in the disease process and can manifest as abdominal pain, malabsorption, nutritional deficiencies, and sarcopenia. T3cDM in chronic pancreatitis is a result of complex endocrine physiology, mainly as a consequence of insulin deficiency from acinar cell fibrosis resulting in reduced production of insulin with resultant hyperglycaemia. Primarily, the islet cell loss affects the beta cells. Other cell types in CP can be affected, including alpha and delta cells as well as PP (pancreatic polypeptide) cells which give CPDM a unique pathophysiology $[5,6]$. As the disease progresses, the diabetes in CP-DM tends to be brittle with impaired glucagon secretion and susceptibility to hypoglycaemia. Diabetes may be the first clinical manifestation of chronic pancreatitis; conversely, it can also be a late complication of chronic pancreatitis.

\section{Diagnosis, Imaging, and Screening}

Distinguishing T3cDM from other aetiologies of diabetes can be challenging. There is no standardised diagnostic criteria for $\mathrm{T} 3 \mathrm{cDM}$ secondary to $\mathrm{CP}$. Type $3 \mathrm{c}$ diabetes is frequently misclassified as type 2 diabetes. Ewald and Hardt have outlined three major criteria for the diagnosis of T3cDM, all of which must be fulfilled: (1) the presence of pancreatic exocrine insufficiency, (2) pathological pancreatic imaging, and (3) the absence of T1DM autoantibodies (Table 1). Minor criteria include impaired $B$ cell function, low levels of fat-soluble vitamins (A, D, E, and K), lack of excessive insulin resistance, and impaired incretin release or pancreatic polypeptide secretion [7]. Typically, the pancreatic polypeptide $(\mathrm{PP})$ response is blunted during mixed meal testing in T3cDM [8].

Morphological evaluation of the pancreas in CP includes abdominal ultrasound and computed tomography (CT), which can be accurate for detecting calcifications (Figure 1) and main pancreatic duct dilation but has low sensitivity for mild to moderate CP changes [9]. MRI and MRCP (and dynamic MRCP with secretin) are typically utilized for the diagnosis of advanced calcified CP, parenchymal atrophy, pseudocysts, and dilation and irregularity of the main pancreatic duct and side branches [10]. In addition to the use

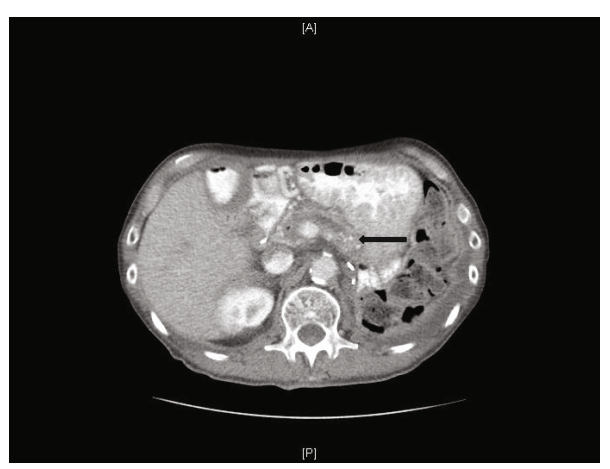

FIGURE 1: CT imaging. Evidence of severe chronic pancreatitis with calcifications (arrow) and dilation of the pancreatic duct.

as an indirect method to predict PEI, endoscopic ultrasound (EUS) is useful in detecting minimal change or noncalcified $\mathrm{CP}$ as well as for detecting and biopsy of pancreatic masses [11]. ERCP is also quite sensitive for CP in addition to its use for interventional options [12].

Risk factors for the development of diabetes in patients with chronic pancreatitis include smoking, longer duration of disease, previous pancreatic surgery, and the presence of calcifications on pancreatic imaging [13-15]. Screening for diabetes (or prediabetes) can be performed annually in patients with chronic pancreatitis and can include fasting and/or random glucose, HbA1c, and OGTT. The diagnostic criteria for the diagnosis of diabetes in patients with chronic pancreatitis are the same as that of T1DM and T2DM; the American Diabetes Association guidelines give values of a fasting plasma glucose of $>7 \mathrm{mmol} / \mathrm{l}$ or higher, a 2-hour plasma glucose level of $11.1 \mathrm{mmol} / 1$ or higher during a $75 \mathrm{~g}$ oral glucose tolerance test, and a random glucose of $11.1 \mathrm{mmol} / \mathrm{l}$ or higher as well as a HbAlc of $>48 \mathrm{mmol} / \mathrm{mol}$ for a diagnosis of diabetes [16]. Functional $B$ cell mass can be measured from serum C-peptide levels during oral glucose tolerance or mixed meal tolerance tests $[17,18]$.

\section{Endocrine Complications}

There is limited data on the rates of hypoglycaemia and ketoacidosis in CP-DM. Hypoglycaemia episodes in CP$\mathrm{DM}$ can be common and are often prolonged due to impaired glucagon secretion. The aetiology of hypoglycaemia in CP$\mathrm{DM}$ is multifactorial and includes the use of insulin, impaired counterregulatory responses, glycogen storage deficits, malnutrition, and malabsorption as well as alcohol consumption [19]. Although long-term data is limited and consensus guidelines on CP-DM patients are lacking, there appears to be a similar risk for micro- and macrovascular complications in comparison to T1DM and T2DM. In the large clinical trials (mainly DCCT and UKPDS), patients with CP-DM were generally excluded. In the only prospective study to date of 54 patients with CP-DM, the risk of diabetic retinopathy was $31 \%$ [20]. In other separate studies, the rate of diabetic retinopathy was $37 \%$, nephropathy was $29 \%$, and peripheral vascular disease was 26\% [21-23]. Chronic pancreatitis is associated with a poor prognosis, with increased morbidity and mortality including an increased risk of pancreatic 
cancer, especially alcoholic pancreatitis, and therefore, longterm complications including retinopathy and nephropathy in some patients do not develop because of reduced life expectancy. In common with T1DM and T2DM, cardiovascular risk factors and lifestyle modifications should be addressed including smoking and alcohol consumption, obesity, hypertension, and hyperlipidaemia. Another important consideration is the development of metabolic bone disease and osteoporosis $[24,25]$; vitamin D testing and supplementation if deficient should be considered in addition to its possible benefit on impaired incretin release in CP-DM [26]. Patients should be monitored and followed up in the same way as T1DM and T2DM for the development of nephropathy, retinopathy, and neuropathy.

\section{Pancreatic Exocrine Insufficiency}

Pancreatic exocrine insufficiency (PEI) is characterised by a deficiency of exocrine pancreatic enzymes which results in malabsorption, malnourishment, and nutrient deficiencies of fat-soluble vitamins. PEI usually precedes DM in chronic pancreatitis but can often be underdiagnosed and undertreated [27]. Malabsorption symptoms include abdominal pain, flatulence, weight loss, and steatorrhea. Malabsorption can be masked by medications, low-fat diets, and poor oral intake. It is estimated that around $80-90 \%$ of patients with CP will have some degree of PEI [28]. Testing for PEI includes the gold standard of the 72-hour faecal fat test which can be time-consuming and impractical for patients, the faecal elastase- 1 test which is not as sensitive in mild to moderate PEI $(200-500 \mu \mathrm{g} / \mathrm{g})$, and the ${ }^{13} \mathrm{C}$-mixed triglyceride $\left({ }^{13} \mathrm{C}-\mathrm{MTG}\right)$ breath test, which is only available in limited centres [29-31]. PERT (pancreatic enzyme replacement therapy) replaces the digestive enzymes that are lost to PEI. PERT is taken in a capsule from typically Creon. The usual starting dose is $40-50,000$ units with meals; a total of $10-25,000$ is taken for snacks [32]. The dose is adjusted until the symptoms of PEI resolve. A referral to a dietitian is essential and is the cornerstone of management in patients with CP. Fatsoluble vitamins may be supplemented if required. PERT has been shown to improve glycaemic control and also to reduce episodes of mild to moderate hypoglycaemia [33].

\section{Pancreatic Cancer}

Around $80 \%$ of pancreatic cancer patients have glucose intolerance or overt diabetes. This association has led to two hypotheses: (1) that pancreatic cancer causes diabetes and (2) that diabetes is a risk factor for the development of pancreatic cancer [34]. Numerous studies have been conducted examining these relationships, with evidence supporting both of these hypotheses; however, the relationship between glucose metabolism and pancreatic cancer remains complex. Chronic pancreatitis and diabetes are both associated with an increased risk of pancreatic cancer, and the degree of hyperglycaemia appears to be related to the risk of developing cancer [35, 36]. There are suggestions that insulin therapy, sulphonylurea therapy, and sitagliptin or exenatide can confer an increased risk of pancreatic cancer. Bonelli et al. found that diabetes was associated with a 2.86 -fold increase in the risk of pancreatic cancer, the risk increasing to 6.49 -fold for those treated with insulin, compared to 2.12-fold for those treated with oral hypoglycaemic agents [37]. Pancreatic cancer is also a cause of diabetes due to unknown mechanisms. Differentiating newonset diabetes as a result of pancreatic cancer may allow for earlier diagnosis and intervention in early-stage disease. Lee et al. have suggested that in distinguishing pancreatic cancer-associated diabetes (type 3cDM) and T2DM, a lack of family history, age 65 years or older, recent weight loss of $>2 \mathrm{~kg}$, and a $\mathrm{BMI}<25 \mathrm{~kg} / \mathrm{m}^{2}$ suggest that type $3 \mathrm{c}$ is more likely [38]. Metformin is used in patients with CP and has been associated with a reduced risk of pancreatic cancer due to its antitumour effects [39]. Studies on the use of biomarkers for screening for pancreatic cancer-associated diabetes are ongoing at present.

\section{Management}

7.1. General Considerations and PERT. Management of CP$\mathrm{DM}$ is challenging and ideally should require multidisciplinary input including a diabetologist, specialist diabetes nurses, diabetes specialist dietitian (preferably with pancreatic experience), and gastrointestinal, hepatobiliary, and pain services [40]. The management of CP-DM can be difficult due to chronic pain, analgesia side effects, nausea, variable nutritional intake, and alcohol consumption. An individualised approach is required, and patients with CP-DM ideally should be best managed in dedicated clinics with clinicians who have an interest and expertise with this condition. Currently, there are no specific guidelines to manage T3cDM in chronic pancreatitis, although there have been recommendations from various consensus groups [41, 42]. Lifestyle modifications include the avoidance of toxic precipitants including smoking and alcohol as well as regular exercise. Specialist dietetic support should be provided with the goals of controlling symptoms of malabsorption and maintaining normoglycaemia. Patients should be encouraged to have a well-balanced diet with no unnecessary fat or carbohydrate restriction. If pain is a constant feature of the disease, referral to a suitable specialist pain clinic is desired. If PEI is present, PERT therapy should be instituted as well as replacement of fat-soluble vitamins if necessary. Screening for the identification of micro- and macrovascular complications should be performed routinely.

7.2. Hypoglycaemic Therapy. Treatment goals will vary between patients and will depend on their diagnosis, nutritional status, lifestyle, and comorbidities. Glycaemic targets include a fasting serum glucose of 3.9-7.2 mmol/l and $\mathrm{HbAlc}$ of $53 \mathrm{mmol} / \mathrm{mol}$; if hypoglycaemic is frequent, glycaemic controls should be relaxed [43]. There are no current guidelines for the use of antihyperglycaemic agents in CP-DM; research into the optimum treatment strategies for type $3 \mathrm{c}$ is lacking. If hyperglycaemia is mild and if insulin resistance is present, metformin should be considered. In addition to its antihyperglycaemic effects, it also can reduce the risk of pancreatic cancer in this population [39]. Adverse side effects 
including weight loss and gastrointestinal upset are undesirable in CP, and patients should be counselled on the potential side effects. Sulphonylureas are associated with hypoglycaemia and are not used as first line therapy in CP-DM. Thiazolidinediones (TZD) are generally avoided due to increased risk of fractures, fluid retention, and heart failure and are not routinely used. Incretin-based therapies (GLP-1 analogs and DPP-IV inhibitors) enhance insulin secretion and have potential in beta cell mass preservation but reduce appetite and can lead to weight loss which is not desired in this population. The possible association with pancreatic cancer and acute pancreatitis would suggest that these medications should not be recommended. There is no data on the use of sodium-glucose cotransporter 2 inhibitors (SGLT2), with the risk of dehydration and weight loss possibly precluding their use [4].

As the main defect in CP-DM is insulin deficiency, for most patients, insulin is the mainstay of treatment and is initiated when oral therapy has not worked or there is uncontrolled hyperglycaemia. Insulin can increase the risk of malignancy in addition to the side effects of weight gain and hypoglycaemia. In advanced CP, basal bolus insulin regimen should be used; consideration should be given to carbohydrate awareness education, continuous glucose monitoring, and insulin pumps in selected patients.

7.3. Autologous Islet Cell Transplantation. Total pancreatectomy with autologous islet cell transplantation (TP-AIT) can relieve pain and preserve beta cell mass in patients with CP when other treatment modalities have failed. It is employed in some centres but with limited numbers and has been shown to improve quality of life and maintain reasonable glycaemic control in certain patients, with benefits seen when the procedure is performed earlier in the disease process, but can be associated with spontaneous hypoglycaemia [44-46].

\section{Summary}

As characterised by our representative patient presented in the clinical vignette, patients with CP-DM tend to have an impaired quality of life, with variable glycaemic control, nutritional deficiencies, and malabsorption. Therefore, the management of CP-DM is challenging and should encompass a multidisciplinary team to deal with short-term and to prevent long-term complications.

\section{Conflicts of Interest}

All authors have no conflict of interest.

\section{References}

[1] N. Ewald, C. Kaufmann, A. Raspe, H. U. Kloer, R. G. Bretzel, and P. D. Hardt, "Prevalence of diabetes mellitus secondary to pancreatic diseases (type 3c)," Diabetes/Metabolism Research and Reviews, vol. 28, no. 4, pp. 338-342, 2012.

[2] Y. Cui and D. K. Andersen, "Pancreatogenic diabetes: special considerations for management," Pancreatology, vol. 11, no. 3, pp. 279-294, 2011.
[3] N. Ewald and R. G. Bretzel, "Diabetes mellitus secondary to pancreatic diseases (type 3c) are we neglecting an important disease," European Journal of Internal Medicine, vol. 24, no. 3, pp. 203-206, 2013.

[4] Y. K. Lin, P. C. Johnston, K. Arce, and B. A. Hatipoglu, "Chronic Pancreatitis and Diabetes Mellitus," Current Treatment Options in Gastroenterology, vol. 13, no. 3, pp. 319-331, 2015.

[5] S. Larsen, J. Hilsted, E. K. Philipsen, B. Tronier, M. D. Nielsen, and $\mathrm{H}$. Worning, "The effect of insulin withdrawal on intermediary metabolism in patients with diabetes secondary to chronic pancreatitis," Acta Endocrinologica, vol. 124, no. 5, pp. 510-515, 1991.

[6] S. Larsen, J. Hilsted, E. K. Philipsen et al., "Glucose counterregulation in diabetes secondary to chronic pancreatitis," Metabolism, vol. 39, no. 2, pp. 138-143, 1990.

[7] N. Ewald and P. D. Hardt, "Diagnosis and treatment of diabetes mellitus in chronic pancreatitis," World Journal of Gastroenterology, vol. 19, no. 42, pp. 7276-7281, 2013.

[8] M. Matsumoto, H. Wakasugi, and H. Ibayashi, "Plasma human pancreatic polypeptide response in chronic pancreatitis," Gastroenterologia Japonica, vol. 17, no. 1, pp. 25-30, 1982.

[9] A. I. De Backer, K. J. Mortelé, R. R. Ros, D. Vanbeckevoort, I. Vanschoubroeck, and B. De Keulenaer, "Chronic pancreatitis: diagnostic role of computed tomography and magnetic resonance imaging," JBR-BTR, vol. 85, no. 6, pp. 304-310, 2002.

[10] S. M. Erturk, T. Ichikawa, U. Motosugi, H. Sou, and T. Araki, "Diffusion-weighted MR imaging in the evaluation of pancreatic exocrine function before and after secretin stimulation," The American Journal of Gastroenterology, vol. 101, no. 1, pp. 133-136, 2006.

[11] T. Stevens, "Role of endoscopic ultrasonography in the diagnosis of acute and chronic pancreatitis," Gastrointestinal Endoscopy Clinics of North America, vol. 23, no. 4, pp. 735747, 2013.

[12] R. Perez-Johnston, N. I. Sainani, and D. V. Sahani, "Imaging of chronic pancreatitis (including groove and autoimmune pancreatitis)," Radiologic Clinics of North America, vol. 50, no. 3, pp. 447-466, 2012.

[13] J. D. Machicado and D. Yadav, "Epidemiology of recurrent acute and chronic pancreatitis: similarities and differences," Digestive Diseases and Sciences, vol. 62, no. 7, pp. 1683-1691, 2017.

[14] G. A. Coté, D. Yadav, A. Slivka et al., "Alcohol and smoking as risk factors in an epidemiology study of patients with chronic pancreatitis," Clinical Gastroenterology and Hepatology, vol. 9, no. 3, pp. 266-273, 2011.

[15] D. Malka, P. Hammel, A. Sauvanet et al., "Risk factors for diabetes mellitus in chronic pancreatitis," Gastroenterology, vol. 119, no. 5, pp. 1324-1332, 2000.

[16] American Diabetes Association, "2. Classification and diagnosis of diabetes," Diabetes care, vol. 39, Supplement 1, pp. S13S22, 2016

[17] G. Cavallini, B. Vaona, P. Bovo et al., "Diabetes in chronic alcoholic pancreatitis. Role of residual beta cell function and insulin resistance," Digestive Diseases and Sciences, vol. 38, no. 3, pp. 497-501, 1993.

[18] R. Lundberg, G. J. Beilman, T. B. Dunn et al., "Early alterations in glycemic control and pancreatic endocrine function in nondiabetic patients with chronic pancreatitis," Pancreas, vol. 45, no. 4, pp. 565-571, 2016. 
[19] L. Mumme, T. G. K. Breuer, S. Rohrer et al., "Defects in $\alpha$-cell function in patients with diabetes due to chronic pancreatitis compared with patients with type 2 diabetes and healthy individuals," Diabetes Care, vol. 40, no. 10, pp. 1314-1322, 2017.

[20] A. Tiengo, T. Segato, G. Briani et al., "The presence of retinopathy in patients with secondary diabetes following pancreatectomy or chronic pancreatitis," Diabetes Care, vol. 6, no. 6, pp. 570-574, 1983.

[21] G. Briani, F. Riva, E. Midena et al., "Prevalence of microangiopathic complications in hyperglycemia secondary to pancreatic disease," The Journal of Diabetic Complications, vol. 2, no. 1, pp. 50-52, 1988.

[22] O. Ziegler, H. Candiloros, B. Guerci, I. Got, T. Crea, and P. Drouin, "Lower-extremity arterial disease in diabetes mellitus due to chronic pancreatitis," Diabetes \& Metabolism, vol. 20, no. 6, pp. 540-545, 1994.

[23] P. A. Hart, M. D. Bellin, D. K. Andersen et al., "Type 3c (pancreatogenic) diabetes mellitus secondary to chronic pancreatitis and pancreatic cancer," The Lancet Gastroenterology \& Hepatology, vol. 1, no. 3, pp. 226-237, 2016.

[24] S. N. Duggan, N. D. Smyth, A. Murphy, D. MacNaughton, S. J. D. O'Keefe, and K. C. Conlon, "High prevalence of osteoporosis in patients with chronic pancreatitis: a systematic review and meta-analysis," Clinical Gastroenterology and Hepatology, vol. 12, no. 2, pp. 219-228, 2014.

[25] S. N. Duggan, M. O’Sullivan, S. Hamilton, S. M. Feehan, P. F. Ridgway, and K. C. Conlon, "Patients with chronic pancreatitis are at increased risk for osteoporosis," Pancreas, vol. 41, no. 7, pp. 1119-1124, 2012.

[26] S. A. Hoogenboom, S. J. Lekkerkerker, P. Fockens, M. A. Boermeester, and J. E. van Hooft, "Systematic review and meta-analysis on the prevalence of vitamin D deficiency in patients with chronic pancreatitis," Pancreatology, vol. 16, no. 5, pp. 800-806, 2016.

[27] J. Keller and P. Layer, "Diagnosis of pancreatic exocrine insufficiency in chronic pancreatitis," in Pancreapedia: Exocrine Pancreas Knowledge Base, 2015.

[28] J. Keller and P. Layer, "Human pancreatic exocrine response to nutrients in health and disease," Gut, vol. 54, suppl_6, pp. 1-28, 2005.

[29] J. Iglesias-Garcia, M. Vilarino-Insua, M. Iglesias-Rey, V. Lourido, and E. Dominguez-Munoz, "Accuracy of the optimized ${ }^{13} \mathrm{C}$-mixed triglyceride breath test for the diagnosis of steatorrhea in clinical practice," Gastroenterology, vol. 124, no. 4, p. A631, 2003.

[30] J. E. Dominguez-Muñoz, C. Hieronymus, T. Sauerbruch, and P. Malfertheiner, "Fecal elastase test: evaluation of a new noninvasive pancreatic function test," The American Journal of Gastroenterology, vol. 90, no. 10, pp. 1834-1837, 1995.

[31] M. O. Othman, D. Harb, and J. A. Barkin, "Introduction and practical approach to exocrine pancreatic insufficiency for the practicing clinician," International Journal of Clinical Practice, vol. 72 , no. 2, article e13066, 2018.

[32] J. E. Domínguez-Muñoz, "Chronic pancreatitis and persistent steatorrhea: what is the correct dose of enzymes?," Clinical Gastroenterology and Hepatology, vol. 9, no. 7, pp. 541-546, 2011.

[33] N. Ewald, R. G. Bretzel, I. G. Fantus et al., "Pancreatin therapy in patients with insulin-treated diabetes mellitus and exocrine pancreatic insufficiency according to low fecal elastase 1 concentrations. Results of a prospective multi-centre trial," Diabe-
tes/Metabolism Research and Reviews, vol. 23, no. 5, pp. 386391, 2007.

[34] F. Wang, M. Herrington, J. Larsson, and J. Permert, "The relationship between diabetes and pancreatic cancer," Molecular Cancer, vol. 2, no. 1, p. 4, 2003.

[35] R. Huxley, A. Ansary-Moghaddam, A. Berrington de González, F. Barzi, and M. Woodward, "Type-II diabetes and pancreatic cancer: a meta-analysis of 36 studies," British Journal of Cancer, vol. 92, no. 11, pp. 2076-2083, 2005.

[36] B. Balkau, E. Barrett-Connor, E. Eschwege, J. L. Richard, J. R. Claude, and P. Ducimetiere, "Diabetes and pancreatic carcinoma," Diabete \& metabolisme, vol. 19, no. 5, pp. 458-462, 1993.

[37] L. Bonelli, H. Aste, P. Bovo et al., "Exocrine Pancreatic Cancer, Cigarette Smoking, and Diabetes Mellitus: A Case-Control Study in Northern Italy," Pancreas, vol. 27, no. 2, pp. 143149, 2003.

[38] J. H. Lee, S. A. Kim, H. Y. Park et al., "New-onset Diabetes Patients Need Pancreatic Cancer Screening?," Journal of Clinical Gastroenterology, vol. 46, no. 7, pp. e58-e61, 2012.

[39] Y. W. Dong, Y. Q. Shi, L. W. He, X. Y. Cui, and P. Z. Su, "Effects of metformin on survival outcomes of pancreatic cancer: a meta-analysis," Oncotarget, vol. 8, no. 33, pp. 5547855488, 2017.

[40] P. D. Hardt, M. D. Brendel, H. U. Kloer, and R. G. Bretzel, "Is pancreatic diabetes (type $3 \mathrm{c}$ diabetes) underdiagnosed and misdiagnosed?" Diabetes Care, vol. 31, Supplement 2, pp. S165-S169, 2008.

[41] M. R. Rickels, M. Bellin, F. G. Toledo et al., "Detection, evaluation and treatment of diabetes mellitus in chronic pancreatitis: recommendations from PancreasFest 2012," Pancreatology, vol. 13, no. 4, pp. 336-342, 2013.

[42] J. E. Dominguez-Munoz, A. M. Drewes, B. Lindkvist et al., "Recommendations from the United European Gastroenterology evidence-based guidelines for the diagnosis and therapy of chronic pancreatitis," Pancreatology, vol. 18, no. 8, pp. 847854, 2018.

[43] S. K. Bhattamisra, T. C. Siang, C. Y. Rong et al., "Type-3c diabetes mellitus, diabetes of exocrine pancreas - an update," Current Diabetes Reviews, vol. 15, no. 5, pp. 382-394, 2019.

[44] P. C. Johnston, Y. K. Lin, R. M. Walsh et al., "Factors associated with islet yield and insulin independence after total pancreatectomy and islet cell autotransplantation in patients with chronic pancreatitis utilizing off-site islet isolation: Cleveland Clinic experience," The Journal of Clinical Endocrinology \& Metabolism, vol. 100, no. 5, pp. 1765-1770, 2015.

[45] R. M. Walsh, J. R. A. Saavedra, G. Lentz et al., "Improved quality of life following total pancreatectomy and auto-islet transplantation for chronic pancreatitis," Journal of Gastrointestinal Surgery, vol. 16, no. 8, pp. 1469-1477, 2012.

[46] Y. K. Lin, C. Faiman, P. C. Johnston et al., "Spontaneous hypoglycemia after islet autotransplantation for chronic pancreatitis," The Journal of Clinical Endocrinology \& Metabolism, vol. 101, no. 10, pp. 3669-3675, 2016. 


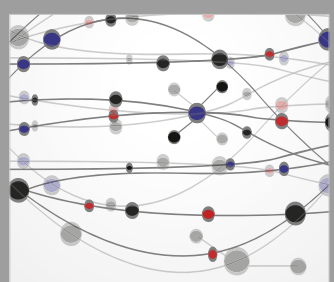

The Scientific World Journal
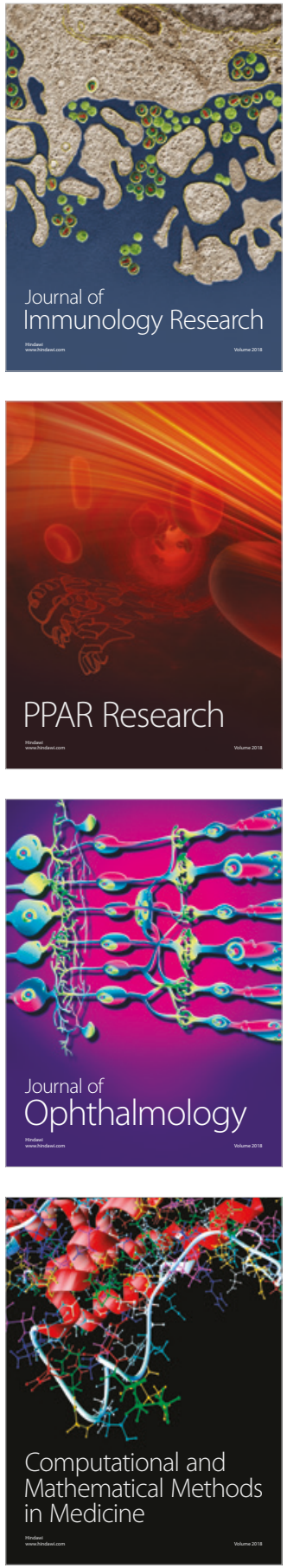

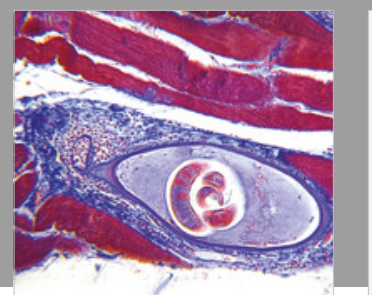

Gastroenterology Research and Practice

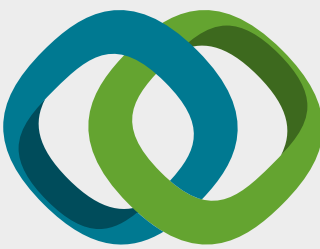

\section{Hindawi}

Submit your manuscripts at

www.hindawi.com
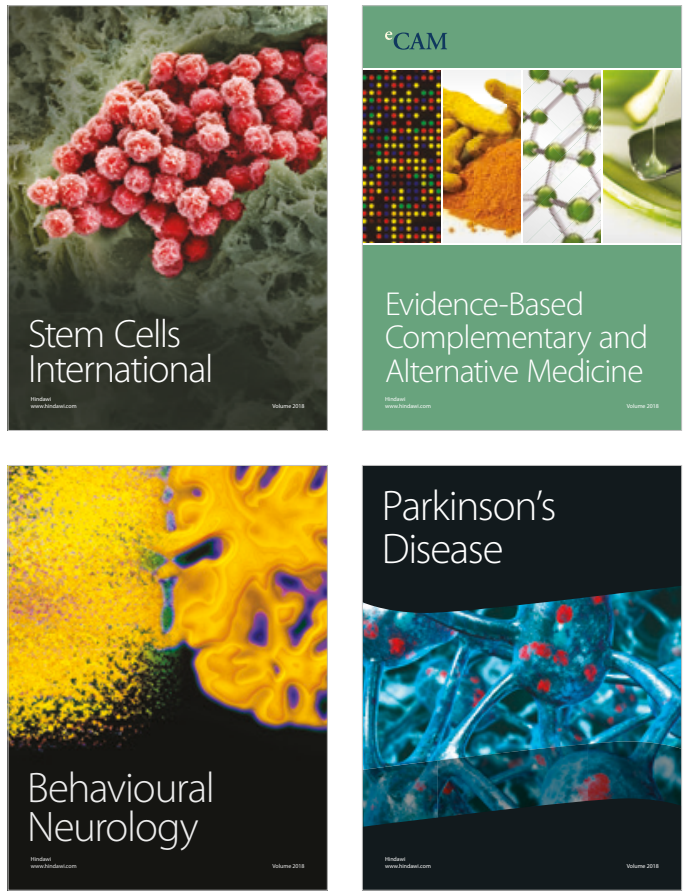

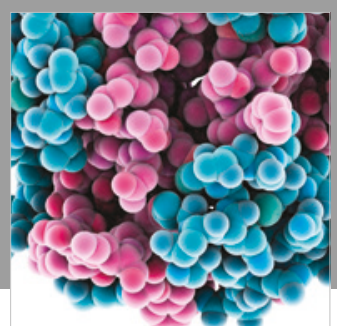

ournal of

Diabetes Research

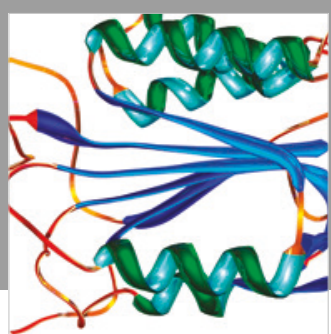

Disease Markers
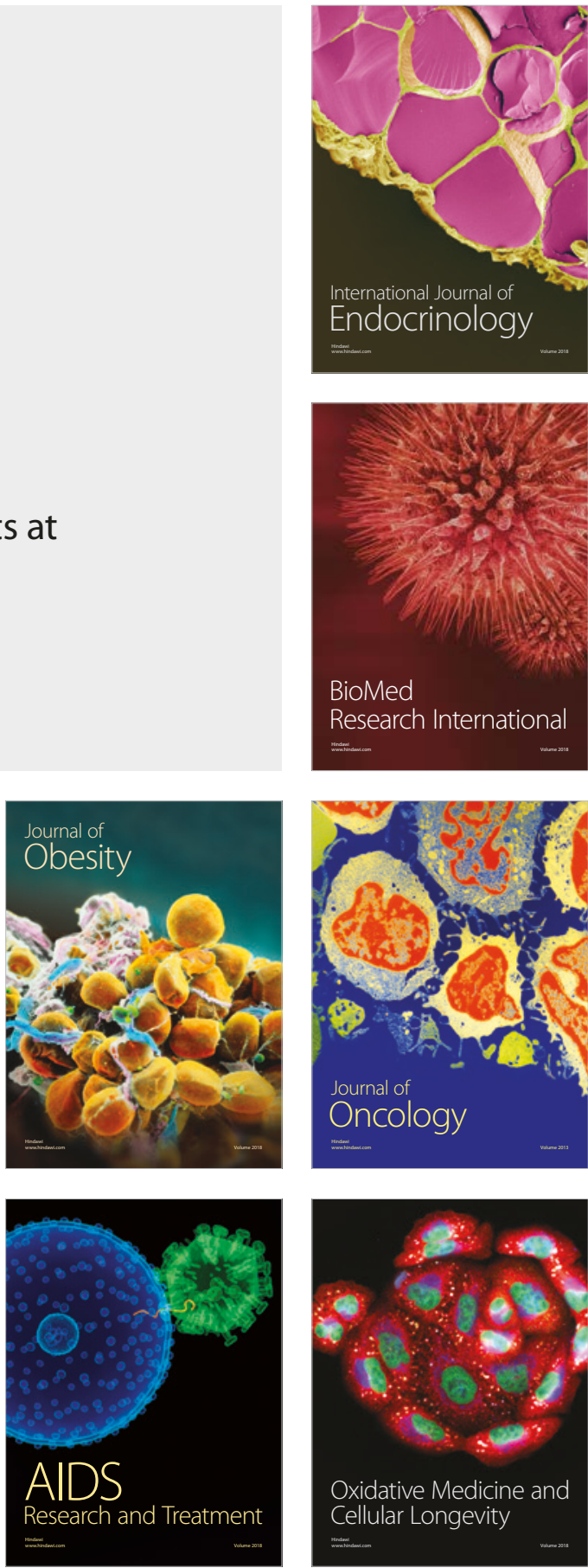